\title{
Is Repeat Testing Needed for Helicobacter pylor?
}

\author{
Robert D. Zipser, MD, and Meghal V. Parikh, MBBS
}

Helicobacter pylori is a major cause of gastritis and gastric and duodenal ulcer disease, and it increases the risk of gastric cancer. Diagnosis and treatment of $H$ pylori infection has become an essential part of the evaluation of patients with dyspepsia and other upper abdominal symptoms. Until recently, diagnosis was made primarily by endoscopic biopsy, and treatment was initiated primarily by gastroenterologists. Several noninvasive office-based diagnostic tests are now readily available to primary care physicians. Measurement of serum antibodies against $H$ pylori is probably the most commonly used noninvasive test, although it does not distinguish active from previous infection. Measurement of the $H$ pylori antigen in the stool and breath tests using urea (labeled with ${ }^{13} \mathrm{C}$ and ${ }^{14} \mathrm{C}$ carbon) are more accurate determinants of active $H$ pylori infection. Because of the availability of noninvasive tests, there is an increased role for the primary care physician in diagnosing and managing this disorder. ${ }^{1}$

The need to retest patients for eradication of the bacteria after treatment has not been established. The success of bacterial eradication depends primarily on patient compliance and on antibiotic resistance, and it varies in different populations. Recurrence rates after confirmed eradication are less than $1 \%$ per year. ${ }^{2}$ In our community we had noted that some of our patients with previously treated $H$ pylori infection who later required repeat endoscopy still had active infection, as determined by endoscopic biopsy. With the recent availability of the low-cost on-site ${ }^{14} \mathrm{C}$-labeled urea breath test, we began testing patients with previously treated $H$ pylori infection to confirm eradication. A retrospective chart review was done to determine whether the incidence of failed treatment warranted recom-

Submitted, revised, 30 December 1999.

From the San Dimas Community Hospital (RDZ, MVP), San Dimas, Calif, and the Department of Medicine (RDZ), UCLA School of Medicine. Address reprint requests to Robert D. Zipser, MD, 1330 West Covina Blvd, Suite 205, San Dimas, CA 91773. mending repeat testing of all patients cared for by our primary care physicians.

\section{Methods}

The patients had been referred to a gastroenterologist (RDZ) for gastrointestinal symptoms and had positive rapid urease testing on endoscopic biopsy within the preceding 3 years. These patients had received a 10 -day course of a proton pump inhibitor twice daily ( $20 \mathrm{mg}$ of omeprazole, or $30 \mathrm{mg}$ of lansoprazole) twice daily, $500 \mathrm{mg}$ of clarithromycin twice daily, and $1 \mathrm{~g}$ of amoxicillin twice daily. If the patient had a history of penicillin allergy, $1 \mathrm{~g}$ of tetracycline twice daily or $500 \mathrm{mg}$ of metronidazole thrice daily was substituted for amoxicillin. Patients were not evaluated for $H$ pylori eradication if they could not cooperate with the testing procedure because of mental impairment or if they had severe medical disorders that made repeat testing inappropriate, such as advanced cancer. Testing was not done for 16 patients who had moved from the area, who did not respond to letters or telephone calls, or who declined to be tested. Six patients deferred testing until they recovered from unrelated illnesses or antibiotic therapy. To date 34 patients, aged 36 to 87 years, were available for testing to confirm eradication of $H$ pylori infection.

The ${ }^{14} \mathrm{C}$-labeled urea breath test was used with on-site analysis at the community hospital. For this noninvasive test, the patient swallows a capsule containing $1 \mu \mathrm{Ci}$ of ${ }^{14} \mathrm{C}$-labeled urea (Ballard Medical Products, Draper, Utah). After 10 minutes, a breath sample is obtained by the patient exhaling through a straw into a balloon. Urease activity in the stomach from $H$ pylori infection releases ${ }^{14} \mathrm{C}$ labeled bicarbonate from the urea, which is then excreted in the breath as ${ }^{14} \mathrm{C}$-labeled carbon dioxide. A negative finding from this test has been validated as an indicator of eradication after treatment by comparison with endoscopic biopsy with a sensitivity of $92 \%$ to $100 \%$, a specificity of $89 \%$ to $100 \%$, a positive predictive value of $91 \%$ to $100 \%$, and a negative predictive value of $98 \%$ to $100 \% .^{3,4}$ 


\section{Results}

One of the 34 patients was excluded from the analysis because her ${ }^{14} \mathrm{C}$-labeled urea breath test result was inconclusive. Five of the remaining 33 patients had positive ${ }^{14} \mathrm{C}$-labeled urea breath tests. All patients claimed that they consumed their medications as directed. All had received omeprazole and clarithromycin; 3 patients also received amoxicillin, 1 received tetracycline, and 1 received metronidazole. The original diagnoses of these 5 patients were duodenal ulcer in 1 , hemorrhagic gastritis in 1, ulcerative esophagitis with erosive gastritis in 1, and erosive gastritis in 2 . Thus, the overall failure rate of treatment in our population of 33 patients is $15 \%$.

\section{Conclusions}

In our patient population that completed treatment for $H$ pylori infection, there was a $15 \%$ incidence of active $H$ pylori infection on repeat testing using the ${ }^{14} \mathrm{C}$-labeled urea breath test. These data are similar to those of several studies that used 10-day triple therapy; after a repeat endoscopy and biopsy to confirm bacterial eradication, they reported a $10 \%$ to $20 \%$ failure rate. ${ }^{5}$

In our community most patients receiving treatment for $H$ pylori infection are evaluated by and receive antibiotic treatment from primary care physicians based on symptoms and positive antibody tests. Health maintenance organizations have encouraged primary care physicians to assume this role in an attempt to reduce unnecessary referrals to specialist physicians. Protocols have been provided to primary care physicians listing noninvasive $H$ pylori testing as part of the initial evaluation of patients with upper abdominal symptoms.

The cost of the ${ }^{14} \mathrm{C}$-labeled urea breath test ranges from $\$ 60$ to $\$ 120$. Considering the incidence of treatment failure and the risks of recurrent ulcers, chronic gastritis, and possibly neoplasm, the cost might be acceptable. With increased patient awareness of this disorder, patients often want eradication confirmed. ${ }^{6}$ The stool antigen test can also be used for this purpose, although some studies show a high rate of false-positive test results. ${ }^{7} \mathrm{Se}-$ rum antibody tests are generally not useful to confirm eradication. Because of the incidence of failed treatment, the low cost of office-based testing, and the need for only one follow-up confirmatory test, we recommend that primary care physicians who treat this disorder provide follow-up evaluation using ${ }^{14} \mathrm{C}$ - or ${ }^{13} \mathrm{C}$-labeled urea breath testing to confirm eradication of $H$ pylori infection.

If treatment has failed to eradicate the infection, we recommend repeat therapy. $H$ pylori infection treatment is sought only by patients who have upper intestinal symptoms. These symptoms are often the result of gastritis, ulcer, or esophageal reflux disease. Eradication of the bacteria promotes healing and markedly reduces the risk of recurrent ulcers and gastritis. Treatment can potentially reduce progression to gastric atrophy, and it can potentially reduce the risk of gastric cancer. Because treatment failures are primarily the result of antibiotic resistance, repeat treatment should be with a different antibiotic regimen.

\section{References}

1. Felz MW, Burke GJ, Schuman BM. Breath test diagnosis of Helicobacter pylori in peptic ulcer disease: a noninvasive primary care option. J Am Board Fam Pract 1997;10:385-9.

2. Abu-Mahfouz MZ, Prasad VM, Santogade P, Cutler AF. Helicobacter pylori recurrence after successful eradication: 5-year follow-up in the United States. Am J Gastroenterol 1997;92:2025-8.

3. Sharma BC, Bhasin DK, Pathak CM, et al. [14C]urea breath test to confirm eradication of Helicobacter pylori. J Gastroenterol Hepatol 1999;14:309-12.

4. Rollan A, Giancaspero R, Arrese M, et al. Accuracy of invasive and noninvasive tests to diagnose Helicobacter pylori infection after antibiotic treatment. Am J Gastroenterol 1997;92:1268-74.

5. Laine L, Suchower L, Frantz J, Connors A, Neil G. Twice-daily, 10-day triple therapy with omeprazole, amoxicillin, and clarithromycin for Helicobacter pylori eradication in duodenal ulcer disease: results of three multicenter, double-blind, United States trials. Am J Gastroenterol 1998;93:2106-12.

6. Fendrick AM, Chey WD, Margaret N, Palaniappan $\mathrm{J}$, Fennerty MB. Symptom status and the desire for Helicobacter pylori confirmatory testing after eradication therapy in patients with peptic ulcer disease. Am J Med 1999;107:133-6.

7. Trevisani L, Sartori S, Galvani F, et al. Evaluation of a new enzyme immunoassay for detecting Helicobacter pylori in feces: a prospective pilot study. Am J Gastroenterol 1999;94:1830-3. 